Check for updates

Cite this: RSC Adv., 2019, 9, 42062

\title{
Functionalization of halloysite nanotubes by enlargement and layer-by-layer assembly for controlled release of the fungicide iodopropynyl butylcarbamate
}

\author{
Xiaobei Jin, (D) Rong Zhang, Minglei Su, (D) Huairui Li, Xianfeng Yue, Daochun Qin* \\ and Zehui Jiang*
}

lodopropynyl butylcarbamate (IPBC) is currently one of the most important fungicides widely used for industrial coatings and bamboo treatment. In this work, a controlled release composite with IPBC for inhibition of mold and stain fungi was prepared using enlarged halloysite nanotubes (HNTs) with layerby-layer (LbL) assembly of polyelectrolyte multilayers. The acid-treated HNTs retained their tubular structure with increased internal diameter, and IPBC loading efficiency was therefore increased to $24.4 \%$, approximately three times the amount of raw HNTs $(8.4 \%)$. In vitro drug release assay showed that IPBC could be released from HNTs in a sustainable manner with a total release amount of $33.8 \%$ over a period of 35 days. The release rate of IPBC could be further controlled by adjusting the number of LbL layers on the tubes and the released amount of IPBC could be limited to less than 10\% in 100 days. An inhibition zone test indicated the as-prepared nanocomposites exhibited significant anti-fungal performance against three mold fungi (Aspergillus niger, Trichoderma viride, and Penicillium citrinum) and one stain fungus (Botryodiplodia theobromae). The results support the potential use of HNTs for a prolonged service life of bamboo products.

Received 19th September 2019 Accepted 15th November 2019

DOI: $10.1039 / c 9 r a 07593 c$

rsc.li/rsc-advances been reported that IPBC only slightly penetrates wood with a inhomogeneous distribution and the amount of IPBC is reduced by approximately $70 \%$ by leaching after 3 months exposure to natural weathering. ${ }^{3}$ Thus, it is important to develop a delivery methodology to gain control over the release profile of the IPBC, as well as reducing the total amount of active agent needed in bamboo protection applications and minimizing the cumulative release to the environment. ${ }^{13}$

Many inhibitor encapsulation systems for reducing the release rate of IPBC have been studied, such as porous silica, polymer microcapsules, sol-gel nanoparticles and other methods. ${ }^{14-17}$ Sørensen et $a l .{ }^{14}$ have synthesized porous silica microparticles containing IPBC to increase the lifetime of the paint film for wood protection. The release of IPBC from paints proved to be prolonged and delayed by use of some types of encapsulated IPBC. Two more studies were published on encapsulating of IPBC within poly(methyl methacrylate) (PMMA) microspheres ${ }^{18}$ and polystyrene microcapsules. ${ }^{19}$ It was found that the release rate of IPBC is highly dependent on the size and morphology of PMMA microparticles, and the experimental polystyrene microcapsules were as effective as the commercial products, despite a slower biocide release rate. The hindered release of IPBC is expected to provide a longer service life for outdoor coatings. Despite of these remarkable advantages of encapsulation systems in drug delivery sectors, most microcapsules reported possess non-uniform 
shapes, have shells of undesirable thickness, lack structural stability, or require complex synthesizing routes, which hinder their wide application. ${ }^{20}$

Compared with these synthetic microcapsules, halloysite nanotubes (HNTs) could be considered as one of the most promising natural material for carrier applications. HNTs are a hydrated polymorph of 1:1 rolling phyllosilicate clay with a chemical formula of $\mathrm{Al}_{2} \mathrm{Si}_{2} \mathrm{O}_{5}(\mathrm{OH})_{4} \cdot n \mathrm{H}_{2} \mathrm{O}$, which is low-cost with rich reserves. ${ }^{21}$ These biocompatible and abundant tubular nanomaterials have an external diameter of $50 \mathrm{~nm}$ to $80 \mathrm{~nm}$, internal lumen of $10 \mathrm{~nm}$ to $50 \mathrm{~nm}$, and length of approximately $1000 \mathrm{~nm} .^{22}$ The hollow structure of HNTs makes it suitable as containers for active molecules. ${ }^{23}$

However, in previous studies investigating the encapsulation of agents in HNTs, the loading efficiency was only approximately $10 \%$ to $15 \%$ because of the limited pore volume of the tube. $^{24}$ In order to increase loading capacity of the HNTs, etching of HNT using acid and alkali treatment were investigated with a target capacity of $40-50 \% .^{25,26}$ Furthermore, due to the negative surface potential in a wide $\mathrm{pH}$ range, it is possible to modify the external surface property of HNTs by adsorbing polycation. ${ }^{27}$ The technique of layer-by-layer (LbL) coated HNTs by polyelectrolytes could significantly control and decrease the diffusion of biocides from the lumen of HNTs, which makes LbL assembly a highly promising approach for long-term bamboo protection.

Although HNTs is a well-known promising carrier for drug delivery without need of expensive and high energy consuming processes, to the best of our knowledge, little information can be seen incorporation of wood fungicide (such as IPBC) into HNTs. In the present study, we described a facile and efficient drug-delivery system by functionalizing of HNTs through acid treatment and coated with LbL polyelectrolyte multilayers for controlled release of IPBC (Fig. 1). The effects of acid treatment on the structure, morphology, and porous characteristics of
HNTs were examined by means of scanning electron microscopy (SEM), transmission electron microscopy (TEM), and $\mathrm{N}_{2}$ absorption/desorption method. The loading efficiency of IPBC in HNTs was investigated with the help of thermogravimetric analysis (TGA). The release behavior was performed to detect its controlled release performance. The ability of the as-prepared nanocomposites to inhibit mold and stain fungi was estimated in laboratory conditions. Concerning future applications, the use of IPBC loaded HNTs in wooden materials appears very promising. Besides sustained drug release, the nanocomposites are proved to achieve the controlled release of IPBC as well as an excellent anti-fungal property.

\section{Experimental section}

\section{Materials}

Halloysite was obtained from Danjiangkou Mineral Factory (Hubei, China). The test fungi, Aspergillus niger, Trichoderma viride, Penicillium citrinum and Botryodiplodia theobromae for this study were purchased from the China Forestry Culture Collection Center. Iodopropynyl butylcarbamate (IPBC), dimethyl sulfoxide (DMSO), ethyl alcohol and hydrochloric acid (HCl) were purchased from Beijing Chemical Reagent Corp. (Beijing, China). Poly(allylamine hydrochloride) (PAH, $M_{\mathrm{w}} \sim$ $10000)$ and sodium poly(styrene sulfonate) (PSS, $\left.M_{\mathrm{w}} \sim 10000\right)$ were purchased from Sinopharm Chemical Reagent Co. Ltd. (Beijing, China). All the chemicals from commercial sources were of reagent grade and used without further purification.

\section{Etching of HNTs}

$\mathrm{HCl}$ solution was selected to dissolve alumina sheets from HNTs. The etching process of HNTs was carried out as follows: $2.5 \mathrm{~g}$ of dry HNTs powder was dispersed into $250 \mathrm{ml}$ of $3 \mathrm{M}(\mathrm{mol}$ $\left.\mathrm{l}^{-1}\right) \mathrm{HCl}$ solution and stirred at $80^{\circ} \mathrm{C}$ for $8 \mathrm{~h}$. Next, the products were centrifuged and washed by deionized water until

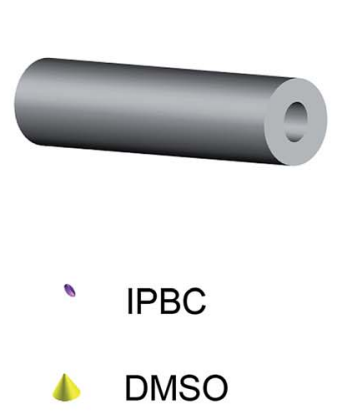

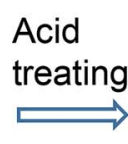

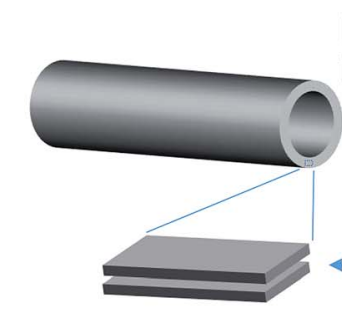

DMSO

intercalating

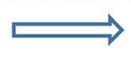

Interlayer

space

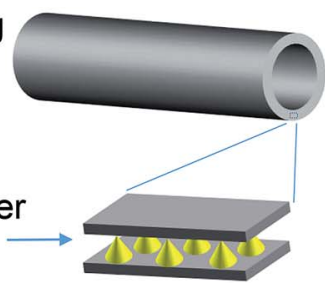

IPBC loading

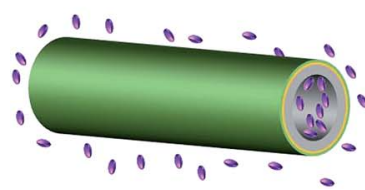

Releasing

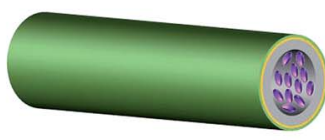

LbL

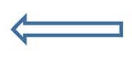

coating
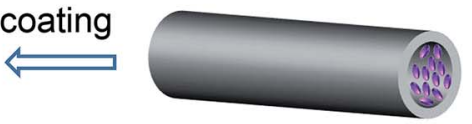

Fig. 1 Schematic illustration of the fabrication of IPBC loaded nanocomposites. 
supernatant becomes neutral. Finally, the acid-etched HNTs were dried in vacuum oven at $60{ }^{\circ} \mathrm{C}$ and then milled to a fine powder, marked as E-HNT.

The surface morphology and microstructure of raw HNTs and acid-etched HNTs were characterized by SEM (FEG-XL30, FEI Company, USA) at a $10 \mathrm{kV}$ accelerating voltage and TEM (TECNAI G2 F30, Philips-FEI, Holland) at $120 \mathrm{kV}$, respectively. The elemental composition was confirmed by energy-dispersive $\mathrm{X}$-ray spectroscopy (EDXS). The surface areas and pore-size distribution of the HNTs were determined by $\mathrm{N}_{2}$ absorption/ desorption measurements (ASAP 2010, Micromeritics Instrument Corp., USA).

\section{IPBC loading procedure}

Before the loading experiments, DMSO as a procedure to improve of the loading capacity of HNTs was developed as previously literature. ${ }^{28}$ The intercalated raw HNTs and acidetched HNTs were marked as HD and E-HD, respectively.

IPBC loading is done as follows: $2.0 \mathrm{~g}$ of HD or E-HD were dispersed into $40 \mathrm{ml}$ of IPBC alcohol solution (500 $\mathrm{mg} \mathrm{ml}^{-1}$ ) and stirred with a magnetic stirrer for $24 \mathrm{~h}$. The resulting mixture was then transferred into a vacuum jar and evacuated using a vacuum pump. During the process of vacuuming, the slight fizzing of the suspension indicated that air in the lumen of HNTs was replaced by IPBC solution. The suspension was kept under vacuum for $30 \mathrm{~min}$ and then cycled back to atmospheric pressure for another $15 \mathrm{~min}$. This process was repeated for three times to increase the loading efficiency. Finally, the solid phase was separated from solution by centrifugation at $5000 \mathrm{rpm}$ for $5 \mathrm{~min}$, washed with ethanol for three times to remove surface adsorbed IPBC molecules and dried at $60{ }^{\circ} \mathrm{C}$ overnight. The IPBC-loaded raw HNTs and acid-etched HNTs were marked as HDI and E-HDI, respectively.

The amount of loaded IPBC in HNTs lumen was determined by the residue from TGA (TA Q500, TA Instruments, USA) at the heating rate of $10{ }^{\circ} \mathrm{C} \mathrm{min}{ }^{-1}$ from 30 to $800{ }^{\circ} \mathrm{C}$ under $\mathrm{N}_{2}$ atmosphere.

\section{LbL assembly}

After loading IPBC, HNTs was coated by LbL technique with PAH/PSS polyelectrolytes. At the following step, the E-HDI samples were immersed in the prepared cationic PAH solution at the concentration of $5 \mathrm{mg} \mathrm{ml}^{-1}$ and continuously stirred for $15 \mathrm{~min}$. Subsequently, the solid samples were centrifuged and washed three times to remove the excess polyelectrolyte. The obtained samples were similarly immersed in anionic PSS solution of the same concentration, centrifuged and washed. The procedure was repeated until desired number of bilayer architecture was reached. 2, 4, and 6 PAH/PSS bilayers on the EHDI were prepared in this study, the corresponding products were marked as E-HDI 2, E-HDI 4 and E-HDI 6 , respectively.

The assembly of polyelectrolyte layers on HNTs was monitored by controlling the surface $\xi$-potential alternation after deposition of each layer using a Zeta Plus Potential Analyzer (Zetasizer Nano ZS, Malvern Instruments Ltd., UK). The microstructure of coating was confirmed by TEM images.

\section{Release characteristics}

The drug release characteristics of IPBC were measured using the dialysis bag technique. A certain amount of IPBC-loaded HNTs (E-HDI, E-HDI 2, E-HDI 4 and E-HDI $_{6}$ ) or free IPBC powder was placed in a dialysis bag. The dialysis bag was immersed into $500 \mathrm{ml}$ of buffer solution $(\mathrm{pH}=7.4)$ at room temperature under magnetic stirring. During the experiments, the concentration of IPBC was always far below the saturation limit to ensure very dilute conditions. ${ }^{\mathbf{1 4}}$

At predetermined time intervals, $5 \mathrm{ml}$ supernatant was collected and replaced with fresh buffer of equal amount, respectively. High performance liquid chromatography (HPLC) was used to determine the concentration of released IPBC in the withdrawn samples. This was quantified at $204 \mathrm{~nm}$ using a seven-points calibration curve of IPBC in the range 1-100 $\mu \mathrm{g}$ $\mathrm{ml}^{-1}\left(R^{2}>0.99\right)$. The tests were carried out in triplicate, and the results were recorded as an average. Cumulative release of IPBC was calculated as follows:

$$
\begin{gathered}
R_{t}=500 C_{n}+\sum_{i=0}^{n-1} C_{i} \\
\text { Cumulative release }(\%)=\frac{R_{t}}{L_{0}} \times 100
\end{gathered}
$$

in which $R_{t}$ indicates the amount of IPBC released at time $t, C_{n}$ represents the $n$th concentration of released IPBC solution, $L_{0}$ is the amount of IPBC initially loaded onto the HNTs.

\section{Inhibition zone test}

The anti-fungal activity of E-HDI ${ }_{4}$ against common bamboo mold (Aspergillus niger, Trichoderma viride, Penicillium citrinum) and stain fungi (Botryodiplodia theobromae) was checked through inhibition zone test. Briefly, mold suspension was applied uniformly on solid potato dextrose agar (PDA) plates, and then one sterilized Oxford cup (outer diameter of $7.8 \mathrm{~mm}$ ) was punched in the center of each plate. $50 \mu \mathrm{l}$ of $\mathrm{E}-\mathrm{HDI}_{4}$ suspension (with $0.5 \%$ IPBC) in ethanol was added in the midpoint of the Oxford cup and equivalent amounts of free IPBC solution in ethanol was used as controls. After 4 weeks of incubation at $25{ }^{\circ} \mathrm{C}$ and $85 \%$ relative humidity, the average diameter of the inhibition zone against fungus surrounding the Oxford cups was measured with a digital caliper using cross method.

\section{Results and discussion}

\section{Characterization of acid-etched HNTs}

HNTs belong to an aluminosilicate group, consist of an internal alumina layer and an external silicate layer, rendering a difference in acid-base properties for both surfaces and affecting the way they interact with solvents. ${ }^{25,29}$

The morphology of HNTs is shown in SEM micrograph (Fig. 2a and b), the raw HNTs show typical tubular structures with a smooth surface. The treatment of $\mathrm{HCl}$ solution resulted in significant alteration in the morphology of HNTs, which exhibits a rough surface with hackly edges on the outer walls. 


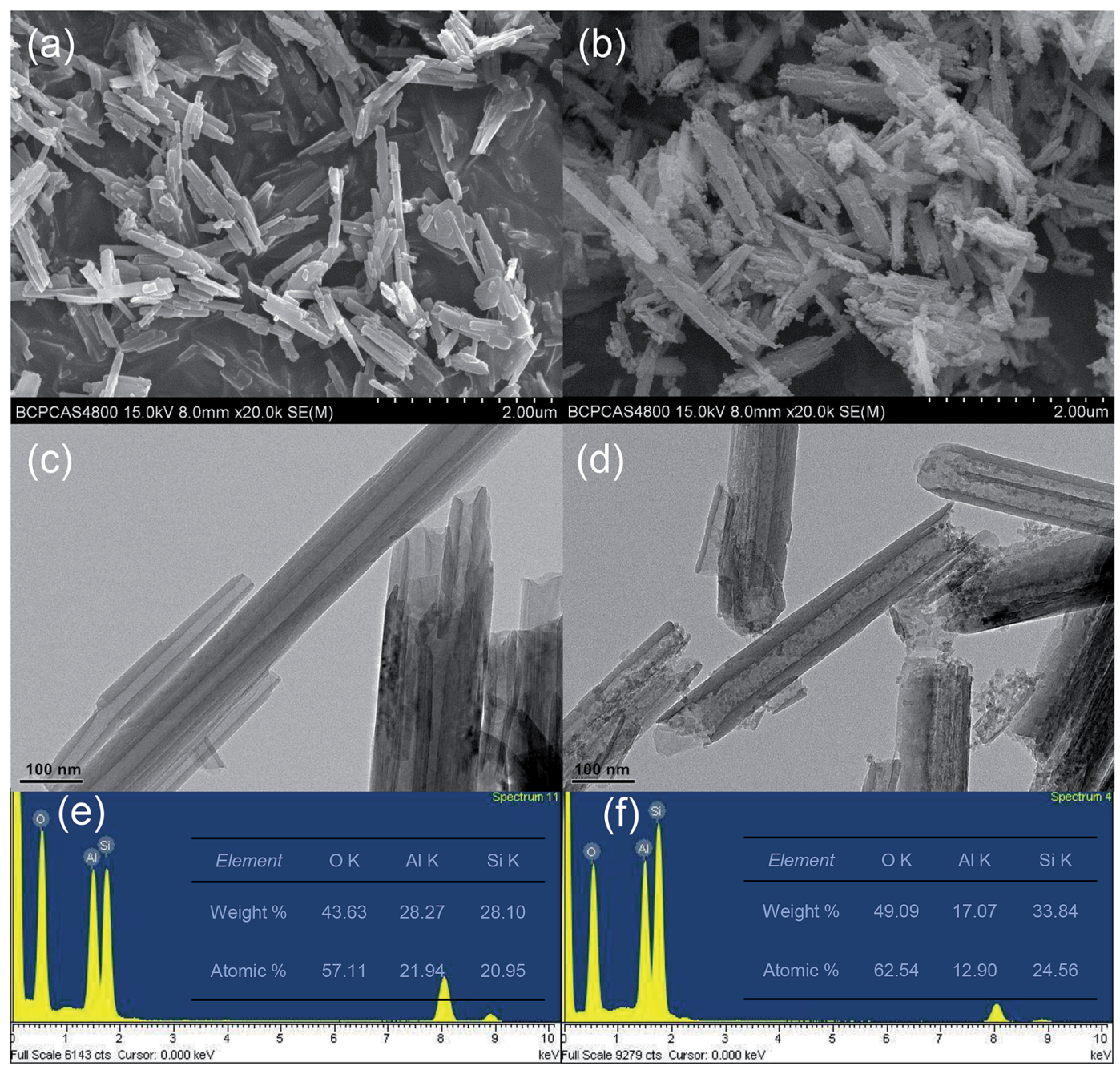

Fig. 2 SEM images, TEM images and EDXS dates of HNTs ( $a, c$ and $e$ ) and acid treated HNTs ( $b$, $d$ and f).

The TEM images in Fig. 2d further reveals that the effect acid treatment on HNTs, leading to small spheroidal nanoparticles filling of most of the nanotubes. These nanoparticles can be found mostly inside the tubes as well as outside, which are considered to be $\mathrm{SiO}_{2}$ originally located in the tube wall. ${ }^{29}$ The acid treatment destroyed the internal alumina sheet, and resulted in the exfoliation of the adjacent silicate sheet. To be noted in TEM images (Fig. 2c and d), the external diameters of the tubes remained unchanged, while the inner diameter increased substantially by the acid-etched samples, indicating that etching takes place solely in the inner lumen of HNTs. ${ }^{30}$ The EDXS mapping of the area suggests that the atomic ratio of $\mathrm{Si} / \mathrm{Al}$ is about $1: 1$ (Fig. 2e), which is close to an ideal value for HNTs. ${ }^{31}$ As shown in Fig. $2 \mathrm{f}$, the overall amount of Al element decreased from $28 \%$ to $17 \%$ with the acid treatment, which can be ascribed to the dissolving of alumina layers. ${ }^{31}$ According to these observations on the structure of raw HNTs and acidtreated HNTs, acid treatment of $3 \mathrm{M} \mathrm{HCl}$ solution resulted an increase in the internal diameter of HNTs, and retained their original tubular-like structure. This is in agreement with previous work, ${ }^{32}$ which reported HNTs etching was optimized depending on time, temperature, and concentration of the acid. The significantly increased lumen volume of HNTs with appropriate etching process would improve the loading efficiency of IPBC.

$\mathrm{N}_{2}$ adsorption and desorption analyses were conducted to investigate the surface area and pore volume of raw HNTs and acid treated HNTs. ${ }^{31}$ As shown in Fig. 3, the isotherm shapes of all samples belong to type IV with a distinct type $\mathrm{H} 3$ hysteresis loop, indicating their preserved mesoporous and microporous structures after acid treatment, ${ }^{32,33}$ which were in accordance with their TEM images. After acid etching, the BET surface area of HNTs (inserted table in Fig. 3) increased from 34.77 to 113.72 $\mathrm{m}^{2} \mathrm{~g}^{-1}$ and the pore volume of HNTs shifted to larger pore sizes $\left(0.45 \mathrm{~cm}^{3} \mathrm{~g}^{-1}\right)$. According to the above morphology study, the raise of the BET surface area and pore volume of acid treated HNTs could be resulted from the dissolution of alumina layers and disaggregation of amorphous $\mathrm{SiO}_{2}$ layers, ${ }^{34}$ indicating the successful development of large porosity in HNTs by acid etching. 


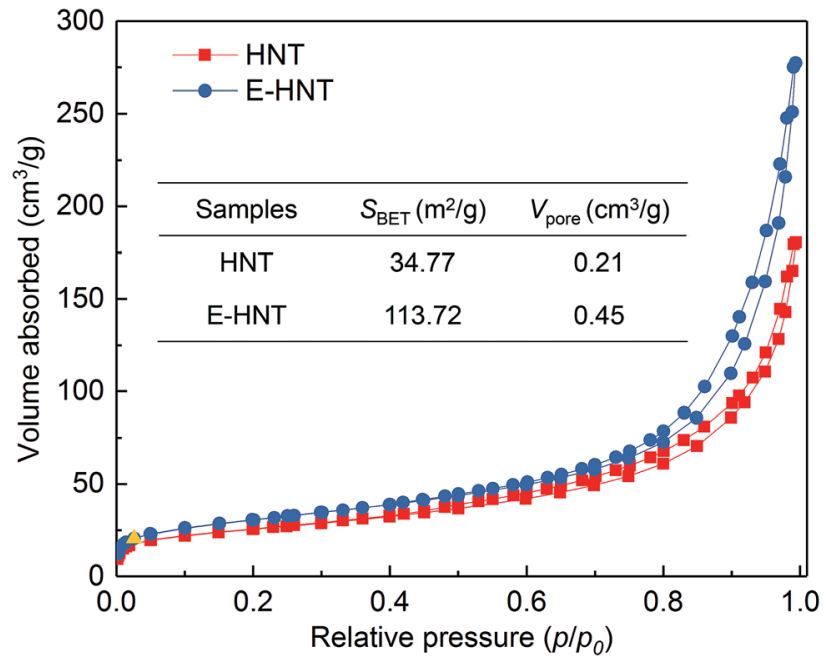

Fig. $3 \quad N_{2}$ adsorption and desorption curves of raw HNTs and acid treated HNTs. The BET surface area and BJH pore volume values are showed in the inserted table.

\section{Thermal properties and IPBC loading}

TGA curves of HNTs, IPBC, IPBC-loaded HNTs (HDI, E-HDI) are displayed in Fig. 4, respectively. As can be seen, the TGA curve of raw HNTs shows a mass loss in a single step in range of 400$600{ }^{\circ} \mathrm{C}$, due to the dehydroxylation of structural alumina groups present in HNTs. ${ }^{35}$ While the TGA curve of HDI shows an additional mass loss in range of $100-240{ }^{\circ} \mathrm{C}$ compare to raw HNTs, due to the thermal degradation of intercalated $\mathrm{DMSO}^{36}$ and incorporated IPBC. According to the weight losses of samples through $100-800{ }^{\circ} \mathrm{C}$, the amount of IPBC loaded in HNTs could be calculated by the following equation:

$$
\mathrm{IPBC} \text { loading }=\frac{R_{\mathrm{HNT}}-R_{\mathrm{HDI}}}{R_{\mathrm{HNT}}-R_{\mathrm{IPBC}}} \times 100 \%
$$

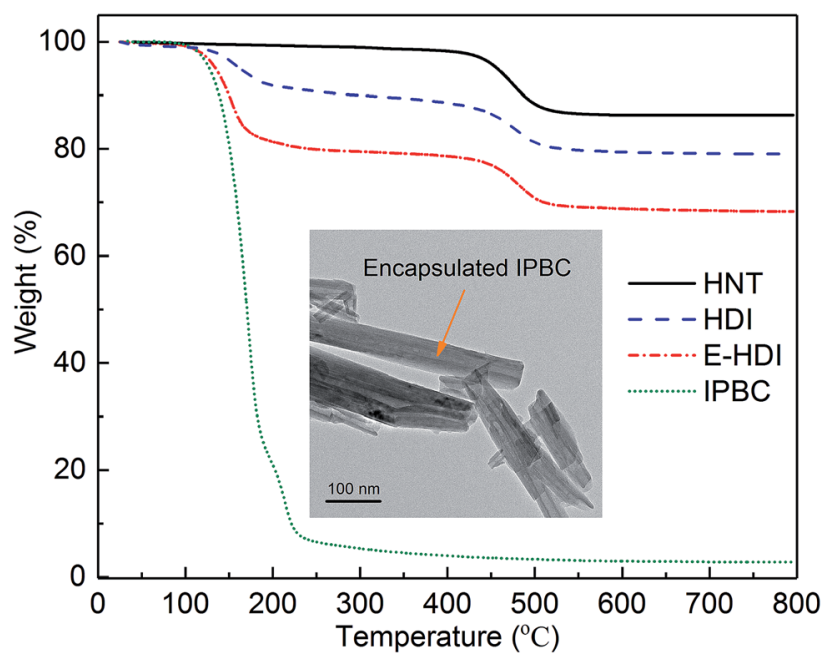

Fig. 4 TGA curves of HNTs, IPBC, HDI and E-HDI. The inset shows TEM image of E-HDI. where $R_{\mathrm{HNT}}, R_{\mathrm{HDI}}$ and $R_{\mathrm{IPBC}}$ are the mass residual of HNTs, HDI and IPBC at $800{ }^{\circ} \mathrm{C}$.

The loading amount of IPBC in the lumen of HD and E-HD were calculated as $8.4 \%$ and $24.4 \%$, respectively. The increase of loading efficiency for IPBC is associated with the increase of the surface area and internal diameter of HNTs after etching removal of alumina by acid treatment.

The successful loading of IPBC into HNTs lumen can also be confirmed by TEM analysis (inserted figure in Fig. 4), in which the hollow space of HNTs was hard to be recognized after loading IPBC. In general, the real distribution of the drug molecules occurs both in the inner lumen along with the outer surfaces of HNTs, but drug bound to the outer-surface was washed out in this study to ensure the effective loading. ${ }^{37}$ Moreover, the enhancement of IPBC loading efficiency in HNTs is an important factor in their industrial application, which can minimize the HNTs consumption and simultaneously avoid the negative effects on coatings or other materials caused by the agglomeration of HNTs nanoparticles. ${ }^{38}$

\section{LbL assembly on HNTs}

Apart from the enhanced loading efficiency, further slowing down drug release time can be performed by coating HNTs with large-molecular-weight polyelectrolyte shells. ${ }^{39}$ HNTs have negative surface, which can be effectively coated by sequential adsorption of positively and negatively charged polyelectrolytes according to the layer by layer (LbL) technique. An earlier paper $^{39}$ has reported the formation of a LbL shell on HNTs successfully by using of PAH as the polycations and PSS as polyanions. Results indicated that with the appropriate molecular weight, the polyelectrolytes can wind around each tube and therefore encapsulate IPBC inside HNTs by blocking the tube ends. The $\xi$-potential of alternating HNTs surfaces for each sequential layer adsorbed is showed in Fig. 5a. There is an obvious symmetric alternation of the surface potential of HNTs corresponding to sequential polycation/polyanion deposition steps, indicating effective LbL assembly.

The TEM images of PAH/PSS shells on HNTs are shown in Fig. 5b-d. It was observed that a coating of LbL layer was evenly covered on the tube surfaces of $\mathrm{E}-\mathrm{HDI}_{2}$, leading to the blurred film at both ends of tube. In the case of 4 bilayers, the flocculent coating of polyelectrolytes by encapsulation of the tubes possessed more tightness, demonstrating that the polymer is winding around the tubes. After coated by 6 bilayers of PAH/ PSS, the aggregation of polymer sealed off the openings of tubes completely. These results are in accordance with that of previous report on the formation of PAH/PSS polyelectrolyte shell on HNTs, which indicated that higher molecular weight polyelectrolytes provide better encapsulations of HNTs. ${ }^{39}$ Usually, the polyelectrolyte shell assembled on the tubes allows thickness tunability in nanoscale. The controlled over permeability can be achieved by optimization of the shell composition, which is inexpensive and effective with a simple waterbased process. ${ }^{\mathbf{4 0 , 4 1}}$ Therefor, in this work, the polyelectrolyte shells on HNTs with tuneable thickness can provide a convenient strategy for controlled release of IPBC. 

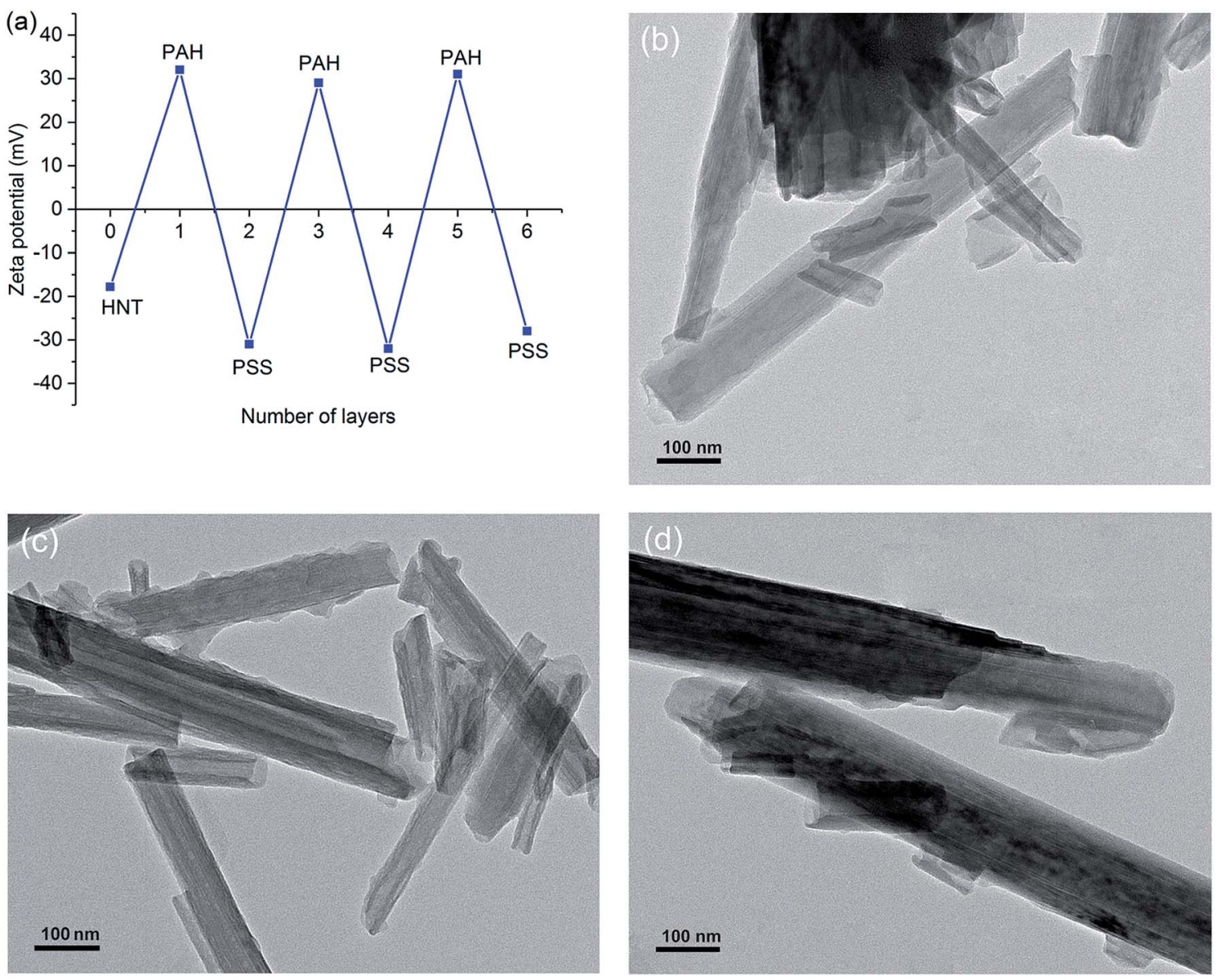

Fig. $5 \xi$-Potential alternation (a) for LbL assembly process of multilayer shells on $\mathrm{HNTS}$ and the corresponding TEM images of E-HDI 2 (b), E- $\mathrm{HDI}_{4}$ (c) and $\mathrm{E}-\mathrm{HDI}_{6}(\mathrm{~d})$.

\section{Release characteristics of IPBC}

The release profiles of IPBC are shown in Fig. 6a. Free IPBC could be released rapidly from a dialysis bag with an initial burst release although its solubility in water is poor. After 5 days, the free IPBC was almost completely released into the water under diluted conditions with a relatively uniform rate, which is ascribed to that the free IPBC was thoroughly contacted with water without the carrier's "protection". In contrast, the EHDI samples showed a relatively sustained drug release profile, only $21.5 \%$ of IPBC released from HDI in 5 days, and the released amount gradually increased to $33.8 \%$ in 35 days, then the release rate gradually slowed down over time. The sustained
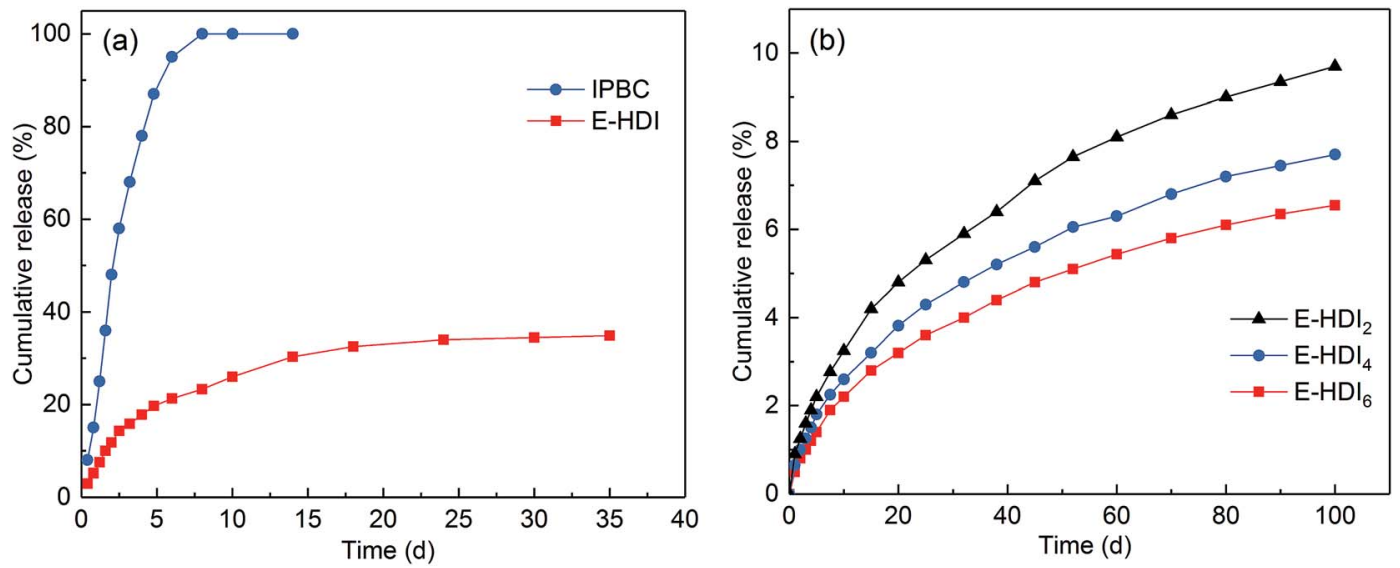

Fig. 6 Release profiles of IPBC from (a) naked E-HDI and (b) coated E-HDI samples in buffer solution. 
drug release may be based on the special lumen of HNTs which confined the IPBC diffusion.

In comparison with that from E-HDI, controllable release of IPBC from tubes was achieved by LbL polyelectrolyte encapsulation of HNTs. As shown in Fig. 6b, the 2, 4, and 6 LbL bilayers encapsulation could further reduced the release rate of IPBC and the released IPBC of 100 days only reached to $9.70 \%, 7.69 \%$ and $6.55 \%$, respectively, which are much slower than the release of IPBC in E-HDI. As can be seen, the release rate of IPBC from polyelectrolyte coated HNTs is in negative correlation with the number of LbL polyelectrolyte layers, therefore it is controllable. The slower release of IPBC is due to the tube architecture of HNTs and polyelectrolyte layers, which can effectively prevent the rapid release of IPBC in water synergistically.

The release kinetics of IPBC from HNTs nanocontainers was evaluated by fitting the release profiles to the Peppas kinetic model $\left(M_{t} / M_{\infty}=k t^{n}\right.$, where $M_{t}$ is the amount of drug released at time $t, M_{\infty}$ is the amount of drug released at infinite time, $n$ is the exponent characteristic of the release mechanism, and $k$ is a constant). ${ }^{42}$ The results showed that the data of E-HDI is well fitted by this model with $n$ equal to $0.44\left(R^{2}>0.99\right)$, indicating a Fickian diffusion mechanism in the cylindrical system. The $n$ values for $\mathrm{E}-\mathrm{HDI}_{2}, \mathrm{E}-\mathrm{HDI}_{4}$ and E-HDI ${ }_{6}$ samples are $0.48,0.48$ and 0.49 , respectively, indicating a non-Fickian diffusion mechanism. It can be concluded that the higher values of $n$ $(0.48,0.48$ and 0.49$)$ are due to the hybrid system of LbL-coated HNTs. That is, the diffusion of IPBC from LbL-coated HNTs is controlled not only by the tube structure of HNTs, but also by polyelectrolyte multilayers, which have a significant influence on the IPBC release rate.

The intensity of the anti-fungal effect would depend on drug concentration released from carriers. ${ }^{19}$ Several studies have been conducted to study the reduced leaching of IPBC in functional capsules. Sørensen et al. ${ }^{\mathbf{1 4}}$ mixed the synthesized porous silica microparticles loaded with IPBC into biocide-free commercial paint matrices. The release of IPBC from paints
Table 1 Inhibition zone diameter for IPBC and $\mathrm{E}-\mathrm{HDI}_{4}$ against mold and blue-stain fungi

\begin{tabular}{lcccc}
\hline & \multicolumn{4}{c}{ Diameter of inhibition zone (mm) } \\
\cline { 2 - 5 } Fungus & IPBC & E-HDI $_{4}$ & E-HD & HNTs \\
\hline \multirow{2}{*}{ Aspergillus niger } & 8.63 & 7.46 & 0 & 0 \\
Trichoderma viride & 20.57 & 18.23 & 0 & 0 \\
Penicillium citrinum & 16.37 & 14.33 & 0 & 0 \\
Botryodiplodia theobromae & 18.97 & 15.66 & 0 & 0
\end{tabular}

proved to level out about $50 \mathrm{~h}$ more than $45 \%$ of the initially encapsulated IPBC. Polystyrene microcapsules containing the biocide IPBC were synthesised and incorporated into coating matrices by Nikkola, ${ }^{19}$ which showed a slower biocide release rate below $10 \%$ during 30 days and lower mould growth in samples of paint. These delayed release of IPBC could possibly be an effect of the thickness and dense of cross-linked silica or polymer shell. However, one drawback of such encapsulation systems is the complex and costly synthesis process. In the case of polyelectrolyte coated HNTs, the hollow structure of HNTs can be easily adjusted by changing the conditions of acid treatment and the controlled released of IPBC can be produced via polyelectrolyte shell structure. Further, the as-prepared nanocontainers with a highly defined structure are also suited to encapsulate various other types of biocides agents for producing durable wooden materials.

\section{Anti-fungal activity of IPBC-loaded nanocomposites}

The inhibition zone represents the area in which the mold growth is stopped due to the inhibitory effect of composites. ${ }^{43}$ Visual images of inhibition zone formed by free IPBC and E$\mathrm{HDI}_{4}$ are presented in Fig. 7. The excellent anti-fungal activity of free IPBC is clearly noticed through maximum inhibition zone of $8.63 \mathrm{~mm}, 20.57 \mathrm{~mm}, 16.37 \mathrm{~mm}$ and $18.97 \mathrm{~mm}$ against Aspergillus niger, Trichoderma viride, Penicillium citrinum and
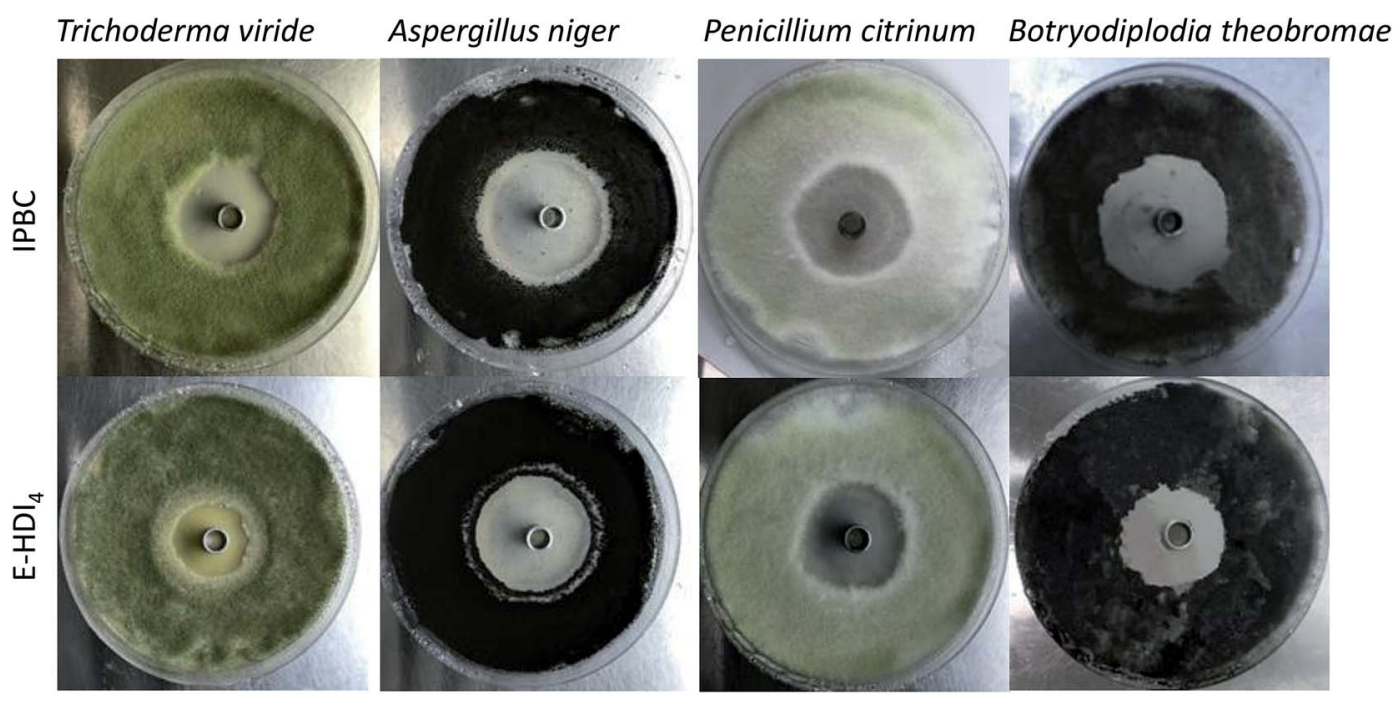

Fig. 7 Photographs of inhibition zone test of IPBC and E-HDI 4 against mold and blue-stain fungi. 
Botryodiplodia theobromae, respectively. While the raw HNTs and E-HD did not display any inhibition zones to mold and stain fungi (Table 1), indicating that the inhibition of mold growth occurred is dependent upon the presence of IPBC ingredients rather than the clays. The E-HDI 4 (with the same amount of free IPBC) also showed good anti-fungal efficiency with inhibition zones of $7.46 \mathrm{~mm}, 18.23 \mathrm{~mm}, 14.33 \mathrm{~mm}$ and $15.66 \mathrm{~mm}$ against Aspergillus niger, Trichoderma viride, Penicillium citrinum and Botryodiplodia theobromae, respectively. However, the diameters of inhibition zone for $\mathrm{E}-\mathrm{HDI}_{4}$ are slightly smaller than free IPBC, which suggest that the sustained release behaviour of IPBC in tubes resulted in a relatively lower IPBC concentration at the same time. It is known that the durability of antifungal effect is a crucial issue in the production of wood protection. In outdoor application, such as in the exposure to continuous raining, the controlled release of IPBC from the HNTs on the surface of paint film is sufficient to maintain the desired biocidal effect. ${ }^{14}$ The hindered release of IPBC could be expected to provide longer protection for the bamboo or other materials. Further more, the on-demand release of IPBC in practical application could be adjusted through varying the composition and number of polyelectrolyte layers as required, ${ }^{\mathbf{4 2}}$ which avoids wasting the drug and polluting the environment.

\section{Conclusions}

In the present study, acid-etched and polyelectrolyte multilayercoated HNTs as a container for controlled release of IPBC were successfully prepared. The HNTs lumen was enlarged by acid treatment, resulting in a higher loading efficiency of IPBC up to 24.4\% (approximately three times as the raw HNTs). The release of IPBC in HNTs was significantly slower than free IPBC, facilitating IPBC release extending to 35 days as seven times as the period of free IPBC. Controlled release of IPBC in HNTs was realized by coating with $\mathrm{LbL}$ polyelectrolyte multilayers, the released IPBC could be limited less than 10\% after 100 days water immersion. The E-HDI ${ }_{4}$ exhibited a good performance in anti-fungal tests, as nearly effective as the free IPBC. This work is believed to promote the understanding of IPBC release characteristics in as-prepared nanocontainers by HNTs and provide a great potential in protection of wood products with a prolonged lifetime.

\section{Conflicts of interest}

The authors declared that they have no conflict of interest.

\section{Acknowledgements}

This work was supported by the National Key Research and Development Program of China (2017YFD0600803) and the Fundamental Research Funds for the International Centre for Bamboo and Rattan (1632019020).

\section{Notes and references}

1 P. Jit Kaur, Forestry Research and Engineering: International Journal, 2018, 2, 240-242.

2 O. Schmidt, D. S. Wei, T. K. H. Tang and W. Liese, J. Bamboo Ratt., 2013, 12, 1-14.

3 T. Volkmer and F. W. M. R. Schwarze, Holz Roh-Werkst., 2008, 66, 181-189.

4 S. Dhyani and D. P. Kamdem, Wood Sci. Technol., 2012, 46, 1203-1213.

5 S. H. Ahn, S. C. Oh, I. G. Choi, G. S. Han, H. S. Jeong, K. W. Kim, Y. H. Yoon and I. Yang, J. Hazard. Mater., 2010, 178, 604-611.

6 Z. G. Sheng, Y. Li, R. M. Fan, X. J. Chao and B. Z. Zhu, Toxicol. Appl. Pharmacol., 2013, 266, 335-344.

7 Y. Liu, P. Laks and P. Heiden, J. Appl. Polym. Sci., 2002, 86, 596-607.

8 H. M. Barnes and G. B. Lindsey, Bioresour. Technol., 2009, 100, 778-781.

9 T. P. Schultz, D. D. Nicholas and A. F. Preston, Pest Manage. Sci., 2007, 63, 784-788.

10 T. Volkmer, H. Landmesser, A. Genoud and F. W. M. R. Schwarze, J. Coat. Technol. Res., 2010, 7, 721-726.

11 L. Juergensen, J. Busnarda, P.-Y. Caux and R. Kent, Environ. Toxicol., 2000, 15, 201-213.

12 Y. F. Li, P. Chen, Y. X. Liu, X. M. Wang and Q. L. Wu, Adv. Mater. Res., 2010, 113-116, 2241-2245.

13 J. M. Pelto, S. Virtanen, T. Munter, J. Larismaa, S. Jamsa and J. Nikkola, J. Microencapsul., 2014, 31, 415-421.

14 G. Sørensen, A. L. Nielsen, M. M. Pedersen, S. Poulsen, H. Nissen, M. Poulsen and S. D. Nygaard, Prog. Org. Coat., 2010, 68, 299-306.

15 T. G. Shutava, R. F. Fakhrullin and Y. M. Lvov, Curr. Opin. Pharmacol., 2014, 18, 141-148.

16 B. G. Trewyn, I. I. Slowing, S. Giri, H. T. Chen and V. S. Lin, Acc. Chem. Res., 2007, 40, 846-853.

17 X. Zeng, B. Zhong, Z. Jia, Q. Zhang, Y. Chen and D. Jia, Appl. Clay Sci., 2019, 171, 20-28.

18 A. Enejder, F. Svedberg, L. Nordstierna and M. Nydén, Proc. SPIE 7903, Multiphoton Microscopy in the Biomedical Sciences $X I, 2011$, p. 79030S.

19 J. Nikkola, Eur. Coat. J., 2014, 4, 36-40.

20 Q. Sun, P. J. Kooyman, J. G. Grossmann, P. H. H. Bomans, P. M. Frederik, P. C. M. M. Magusin, T. P. M. Beelen, R. A. van Santen and N. A. J. M. Sommerdijk, Adv. Mater., 2003, 15, 1097-1100.

21 E. Joussein, S. Petit, J. Churchman, B. Theng, D. Righi and B. Delvaux, Clay Miner., 2005, 40, 383-426.

22 Y. Lvov and E. Abdullayev, Prog. Polym. Sci., 2013, 38, 16901719.

23 E. Abdullayev and Y. Lvov, J. Nanosci. Nanotechnol., 2011, 11, 10007-10026.

24 M. H. Lee, H.-S. Seo and H. J. Park, J. Food Sci., 2017, 82, 922932.

25 R. D. White, D. V. Bavykin and F. C. Walsh, Nanotechnology, 2012, 23, 065705. 
26 T. Gaaz, A. Sulong, A. Kadhum, M. Nassir and A. Al-Amiery, Materials, 2016, 9, 620.

27 D. G. Shchukin, S. V. Lamaka, K. A. Yasakau, M. L. Zheludkevich, M. G. S. Ferreira and H. Molhwald, J. Phys. Chem. C, 2008, 112, 958-964.

28 Z.-L. Cheng, B.-C. Cao and Z. Liu, Micro Nano Lett., 2019, 14, 585-589.

29 E. Abdullayev, A. Joshi, W. Wei, Y. Zhao and Y. Lvov, ACS Nano, 2012, 6, 7216-7226.

30 D. Garcia-Garcia, J. M. Ferri, L. Ripoll, M. Hidalgo, J. LopezMartinez and R. Balart, Appl. Surf. Sci., 2017, 422, 616-625.

31 A.-B. Zhang, L. Pan, H.-Y. Zhang, S.-T. Liu, Y. Ye, M.-S. Xia and X.-G. Chen, Colloids Surf., A, 2012, 396, 182-188.

32 L. Deng, P. Yuan, D. Liu, P. Du, J. Zhou, Y. Wei, Y. Song and Y. Liu, Appl. Clay Sci., 2019, 181, 105240.

33 D. Papoulis, S. Komarneni, A. Nikolopoulou, P. TsolisKatagas, D. Panagiotaras, H. G. Kacandes, P. Zhang, S. Yin, T. Sato and H. Katsuki, Appl. Clay Sci., 2010, 50, 118-124.

34 C. Li, Y. Zhao, T. Zhu, Y. g. Li, J. Ruan and G. Li, RSC Adv., 2018, 8, 14870-14878.
35 G. Gorrasi, Carbohydr. Polym., 2015, 127, 47-53.

36 N. Mahrez, S. Bendenia, K. Marouf-Khelifa, I. BatonneauGener and A. Khelifa, Compos. Interfaces, 2015, 22, 403-417.

37 Y. Lvov, W. Wang, L. Zhang and R. Fakhrullin, Adv. Mater., 2016, 28, 1227-1250.

38 M. Makaremi, P. Pasbakhsh, G. Cavallaro, G. Lazzara, Y. K. Aw, S. M. Lee and S. Milioto, ACS Appl. Mater. Interfaces, 2017, 9, 17476-17488.

39 N. G. Veerabadran, D. Mongayt, V. Torchilin, R. R. Price and Y. M. Lvov, Macromol. Rapid Commun., 2009, 30, 99-103.

40 A. A. Antipov and G. B. Sukhorukov, Adv. Colloid Interface Sci., 2004, 111, 49-61.

41 K. Ariga, J. P. Hill, M. V. Lee, A. Vinu, R. Charvet and S. Acharya, Sci. Technol. Adv. Mater., 2008, 9, 014109.

42 E. Ruiz-Hitzky, K. Ariga and Y. M. Lvov, Bio-inorganic Hybrid Nanomaterials: Strategies, Syntheses, Characterization and Applications, 2007, pp. 419-441.

43 R. Rajiv Gandhi, S. Gowri, J. Suresh and M. Sundrarajan, J. Mater. Sci. Technol., 2013, 29, 533-538. 